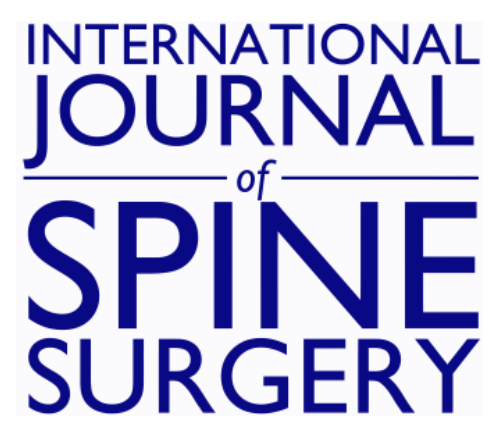

\title{
Thoracic Endoscopic Spine Surgery: A Comprehensive Review
}

\author{
BRIAN FIANI, IMRAN SIDDIQI, TAYLOR REARDON, KASRA SARHADI, ALEXANDER \\ NEWHOUSE, BRANDON GILLILAND, CYRUS DAVATI and AKASH VILLAIT
}

Int J Spine Surg 2020, 14 (5) 762-771

doi: https://doi.org/10.14444/7109

http://ijssurgery.com/content/14/5/762

This information is current as of April 26, 2023.

Email Alerts Receive free email-alerts when new articles cite this article. Sign up at: http://ijssurgery.com/alerts 


\title{
Thoracic Endoscopic Spine Surgery: A Comprehensive Review
}

\author{
BRIAN FIANI, DO,${ }^{1}$ IMRAN SIDDIQI, DO,${ }^{2}$ TAYLOR REARDON, DO,${ }^{3}$ KASRA SARHADI, MD, MPH, ${ }^{4}$ \\ ALEXANDER NEWHOUSE, BS, ${ }^{5}$ BRANDON GILLILAND, DO,${ }^{6}$ CYRUS DAVATI, DO,${ }^{7}$ AKASH VILLAIT, \\ $\mathrm{DO}, \mathrm{MS}^{8}$ \\ ${ }^{1}$ Desert Regional Medical Center, Palm Springs, California, ${ }^{2}$ Western University of Health Sciences College of Osteopathic Medicine, Pomona, California, \\ ${ }^{3}$ University of Pikeville, Kentucky College of Osteopathic Medicine, Pikeville, Kentucky, ${ }^{4}$ Miller School of Medicine, University of Miami, Miami, Florida, ${ }^{5}$ Rush \\ University Medical Center, Chicago, Illinois, ${ }^{6}$ Alabama College of Osteopathic Medicine, Dothan, Alabama, ${ }^{7}$ New York Institute of Technology College of \\ Osteopathic Medicine, Glen Head, New York, ${ }^{8}$ Midwestern University, Arizona College of Osteopathic Medicine, Glendale, Arizona
}

\begin{abstract}
Background: From the 1990s, there has been growth in the literature demonstrating the feasibility of minimally invasive approaches for treating diverse spinal disorders. There is still much work to be done in circumnavigating the technical challenges and elucidating relative advantages of endoscopic techniques in spine surgery. In this comprehensive literature review, we discuss the history, advantages, disadvantages, approaches, and technology of, and critically examine peer-reviewed studies specifically addressing, endoscopic thoracic spinal surgery.

Methods: Literature review was conducted with the key words "endoscopic," "minimally invasive," and "thoracic spinal surgery," using PubMed, Web of Science, and Google Scholar.

Results: Review of 241 thorascopic procedures showed a success rate of $98 \%$ to $100 \%$, low morbidity, and favorable complication profile. Review of 115 thoracic fixation procedures demonstrated high success rate, and $87 \%$ of screw positions were rated "good." Review of 55 full endoscopic uniportal decompressions showed sufficient decompression in most patients. Match pair analysis of 34 patients comparing video-assisted thoracoscopy surgery (VATS) or posterior spinal fusion reported the VATS group had increased operative duration but reduced blood loss.

Conclusions: Based on our literature review, there is a high rate of positive outcomes with endoscopic thoracic spine surgery, which reduces tissue dissection, intraoperative blood loss, and epidural fibrosis. However, the technical challenge highlights the importance of further training and innovation in this rapidly evolving field.

Level of Evidence: 3.

Clinical Relevance: There is growing evidence demonstrating the success of endoscopic thoracic spinal surgery. Populations that could be helped include the elderly and immunocompromised, who would benefit from decreased hospital stay and enhanced recovery time.

Endoscopic

Keywords: endoscopic, minimally invasive, thoracic spine
\end{abstract}

\section{INTRODUCTION}

Thoracic endoscopic spine surgery is a form of minimally invasive spine surgery (MISS) primarily used to treat thoracic spinal disc herniations and stenosis via endoscopic discectomies and decompression techniques, respectively. ${ }^{1}$ Because of the lengthy recovery time and associated complications of open procedures, this minimally invasive approach primarily aims to reduce tissue trauma while retaining therapeutic efficacy. ${ }^{1}$

Historically, endoscopic spine surgery is a relatively new field that has been developing for the last 40 years. Although the technique was initially used to treat lumbar disc herniations, developments in camera, drill, and endoscope technology have opened up new horizons. The first endoscopic spine surgeries used the percutaneous nucleotomy technique, which only enabled the use of small needlelike instruments. A breakthrough was made in 1990 when Parvis Kambin delineated a triangular safe zone, later known as Kambin's triangle. This triangular zone is bordered by the exiting root anteriorly, the traversing root medially, and the superior end plate of the lower lumbar vertebra inferiorly. Kambin's triangle not only enabled the introduction of larger surgical instruments into the field, but also facilitated access to foraminal pathology. As technology progressed, Parvis Kam- 
bin's discovery paved the way for various procedures in the lumbar region of the spine. ${ }^{2}$

With regard to the development of thoracic endoscopic spine surgery, adaptations of thoracic surgical techniques like thoracoscopies and videoassisted thoracoscopy surgeries (VATS) were first used in the early 1990s. However, the morbidity and steep learning curve associated with approaching the thoracic spine via the chest cavity has prompted the development of more MISS techniques with a posterior or posterolateral approach. Evaluating these MISS techniques for the thoracic spine will be the focus of this review. ${ }^{2}$

\section{ADVANTAGES}

Selection of the proper approach by neurosurgeons is determined by anatomic location, consistency of the pathology, the general condition of the patient, and the surgeon's experience. ${ }^{3}$ Use of endoscopic techniques in the thoracic spine has proven to be efficacious for surgical aptitude and patient outcomes. An endoscopic approach, compared with traditional open spine surgery, shows the general benefits of less tissue dissection, muscle trauma, intraoperative blood loss, damage to epidural blood supply, and epidural fibrosis. ${ }^{4}$ Endoscopic approaches have also been used by neurosurgeons to improve the visualization of spinal pathologies. A study performed by $\mathrm{Ahn}^{1}$ on the current techniques of endoscopic decompression in spine surgery found that endoscopic visualization provides the neurosurgeon with a wider visual field compared with a microscopic visualization. Ruetten et $\mathrm{al}^{3}$ noted the visual field benefits of endoscopic approaches in disc herniations and stenosis of the thoracic spine, emphasizing that a nonanterior approach may involve difficult or inadequate visualization or handling of the area anterior to the spinal cord and approach-related destabilization of the posterior structures.

Endoscopic techniques used in thoracic spine surgery have improved patient outcomes by reducing hospital stay duration, and providing earlier functional recovery and overall improvement in quality of life. ${ }^{4}$ A reduction in hospital stay duration can be correlated with a reduction in potential for nosocomial infection acquisition. Patients also benefit from endoscopic approaches intraoperatively. The endoscopic spine decompression techniques outlined thoroughly by $\mathrm{Ahn}^{1}$ emphasize the use of only general or epidural anesthesia or conscious sedation, which can lead to the aforementioned benefits. As pointed out in a study by Lin et $\mathrm{al},{ }^{5}$ patients who are elderly, who are immunocompromised, or those that have multiple comorbidities would benefit from minimally invasive surgery. This can be applied to patient selection in thoracic spine surgery cases because there is a lower chance of exacerbation of these conditions with an endoscopic approach.

\section{DISADVANTAGES}

When considering the use of endoscopic approaches to thoracic spine surgery, the surgeon must also be aware of the disadvantages as much as, if not more than, the advantages. Use of the technique has been documented in thoracic spine cases but pales in comparison to the extensive documentation of endoscopic approaches in lumbar and cervical spine cases. A lack of published clinical data can influence a neurosurgeon to not use that technique.

A glaring disadvantage in using the endoscopic approach in thoracic spine surgery is the technical challenges that accompany it. The learning curve to master the technique is relatively long and challenging before clinical success without complications can be ensured. ${ }^{1}$ Even with proficiency in the technique, technical challenges can be seen based on the type of procedure being performed. Bae et $\mathrm{al}^{6}$ studied the use of percutaneous endoscopic thoracic discectomy and found that this procedure can be technically challenging in the surgical treatment of upper and mid thoracic disc herniations because of the complexity of neural and vascular structures. Orientation-related technical hurdles were demonstrated in the same study because a posterior thoracic endoscopic approach offered the surgeon a higher manual dexterity, anatomic familiarity, direct approach to ventral epidural space, and little approach-related morbidity, as opposed to an anterior approach, which involves dissection around risky anatomic structures, general anesthesia, 1-lung ventilation, and nerve root retraction. ${ }^{6}$ It can also be noted that with increased technicality comes the need for not only an experienced surgeon but an experienced assistant and surgical team. ${ }^{7}$ Patient safety must not be sacrificed when examining the disadvantages of using endoscopic approaches in thoracic spine surgery. 


\section{INDICATIONS}

A thorough neurosurgical workup can provide the surgeon with indications of using endoscopic approaches to the thoracic spine. It should be considered in elderly patients or patients with comorbidities because it is often performed under local or regional anesthesia. ${ }^{1}$ An endoscopic approach to thoracic spine surgery should also be considered if the patient is concerned with cosmetic consequences, because endoscopy promotes better cosmesis.

Adequate and recent diagnostic studies, such as spinal magnetic resonance imaging (MRI) or computed tomography (CT) no more than 3 months prior to operation, should be obtained as diagnostic standards for establishing indicated interventions. ${ }^{8}$ Traditionally, surgery is indicated for a neurologic deficit, structural deformity, or nonsurgical treatment failure. ${ }^{5}$ Endoscopic approaches have been documented in numerous thoracic spine pathologies and are even the gold standard approach in microsurgical microscopic disc surgery, also known as "microdiscectomy." 8 Evidence compiled by Ruetten et $\mathrm{al}^{3}$ shows that interlaminar, extraforaminal, or transthoracic retropleural approaches in full-endoscopic uniportal decompressions in the thoracic spine allow for sufficient decompression, minimize trauma, and have technical advantages and low complication rates. Cadaver studies performed by Abuzayed et $\mathrm{al}^{7}$ indicated that endoscopic approaches would allow for better visualization of the anterior thoracic spine, which can improve quality of treatment in conditions such as herniated discs, vertebral body instability from trauma/degeneration, infective lesions affecting the spine/disc space, and even thoracic sympathectomy. The improved visualization of the ventral thoracic spine is also shown to provide benefits in separation surgery in thoracic metastases, which could decrease the need to perform a costotransversectomy. ${ }^{9}$ It should be reemphasized that proper use and adaptation of the techniques in the thoracic spine can be technically challenging and should only be considered if patient safety remains uncompromised.

\section{CONTRAINDICATIONS}

There is a noticeable degree of overlap between the aforementioned disadvantages of endoscopic techniques in thoracic spine surgery and contrain- dications for its use. Because of the degree of technical difficulty and necessary training required to perform it, the surgeon must not force it into their repertoire. General contraindications for endoscopy in spine surgery, as outlined by Birkenmaier et $a l,{ }^{8}$ include but are not limited to: cauda equina syndrome, clinically relevant instabilities, deformities or back pain that is not due to neural compression, and very large disc herniations with or without a fresh motor deficit.

Most contraindications are characteristic of the procedure being performed. For example, it has been noted that endoscopic decompression techniques in the thoracic spine are not suitable for conditions such as segmental instability, tumors, trauma, infection, and deformities. ${ }^{1}$ In certain circumstances, calcified discs, severe stenosis, painless weakness, or severe fibrotic tissue adhesion can also be contraindications in endoscopic approaches. ${ }^{1}$ An integration of knowledge of neurosurgical interventions and endoscopic adaptations is necessary when assessing the contraindications of a specific case.

\section{THORACIC VENTRAL ENDOSCOPIC APPROACH}

The focus of this review is not to elucidate the technical details involved in specific procedures using an endoscopic approach to the ventral spine, but to provide an overview of its general principles and utility. In 1992, John Regan and Daniel Rosenthal independently developed thoracoscopic spinal surgery. ${ }^{10}$ One method to access the thoracic spine that has been developed since then is the ventral endoscopic approach. Before the introduction of endoscopic approaches, open ventral approaches in thoracic spine surgery were preferred when attempting to access pathology more directly and to prevent disruption of posterior structures. ${ }^{11}$ This approach could be employed in order to address a diverse array of pathologies, including neoplasm, herniated discs, vertebral body instability, or infection. ${ }^{12}$ However, when addressing anterior spinal column pathologies using a traditional open thoracotomy, large exposure windows up to $20 \mathrm{~cm}$ are used with resulting complications that can include intercostal neuralgia, postthoracotomy syndromes, and increased morbidity. ${ }^{13}$ With the advent of the thoracic ventral endoscopic approach, surgical goals, such as neural decompression, vertebral reconstruction, and screw plate 
fixation, could be achieved while avoiding the higher risk of complications posed by open approaches.

In an overview of the technique involved with a ventral endoscopic approach to thoracic spine surgery, one must begin by considering which side of the patient should be used for port placement. In general, the thoracic spine is easier to access through the right chest wall because this surgical corridor avoids such structures as the heart and aorta. ${ }^{7}$ For a right-sided approach, the patient is placed in left lateral decubitus position, with the ipsilateral arm in an airplane sling, abducted away from the chest wall. The patient's chest is prepped and draped in the usual sterile fashion, and the chest wall is entered near the midaxillary line, with subsequent passage of endoscopic ports. These ports are used to pass surgical instruments into the chest wall, depending on the specific case. ${ }^{14}$ One port is used for the endoscope, with the others being reserved for working instruments. Additionally, an endoscopic lung retractor is used to protect the lungs from instruments. ${ }^{15}$ In the specific case of discectomy, the patient is positioned in the lateral decubitus position, with the working port placed over the affected disc, along the posterior axillary line. The camera port is positioned in the midaxillary line, caudal to the working port. The rib head is removed and the lateral surface of the pedicle as well as neural foramen are exposed. The pedicle and floor of the spinal canal are resected in order to decompress the ventral spine. For corpectomy, the same position and port locations are used, the segmental vessels are divided, and the adjacent discs above and below the targeted corpus are removed. The ipsilateral pedicle is removed to decompress the anterior cord, followed by median corpectomy. ${ }^{7}$

Achieving success with this advanced and innovative ventral endoscopic approach not only requires the surgeon to have extensive familiarity with open ventral spine anatomy, but also presents novel technical challenges. For example, the surgeon must gain new skills in so-called tubology and scopology, which encompasses maintaining clean ports and scopes, retaining scope stability, orienting the scope effectively, navigating appropriately without tactile feedback, and adapting to monocular vision without depth perception. ${ }^{16}$ Despite the aforementioned hurdles, innovations in instrumentation have been easing the learning curve, making thoracic ventral endoscopic approaches more feasible. Moreover, ventral endoscopic approaches present clear benefits compared with traditional open approaches, including fewer intraoperative and postoperative complications, a decreased need for pain medications, as well as shorter hospital stays. ${ }^{10}$ Additionally, ventral endoscopic approaches have been shown to be as effective as open approaches in achieving surgical success. A more in-depth comparison between traditional open approaches and endoscopic approaches will be discussed in later sections of this article.

\section{THORACIC DORSAL AND DORSOLATERAL ENDOSCOPIC APPROACH}

Most traditional open thoracic spine surgeries use a dorsal approach. One of the primary reasons for a dorsal approach in an open technique is to allow the operator direct access to the bony elements and spinal canal. However, as in the ventral approach, an open dorsal approach carries significant risk, most notably iatrogenic collateral damage to retracted paraspinal musculature and neurovascular structures, including the spinal cord itself. Some examples of common complications include epidural fibrosis and "fusion disease" (a term referring to the disruption/damage of the posterior extensor musculature precipitated by the dissection and retraction of this musculature). The advantage of an endoscopic dorsal approach to the thoracic spine is that there is less risk of damage to structures given the use of minimally invasive ports. When accessing the spinal canal, an endoscopic technique also requires a smaller laminotomy than it would in an open approach. ${ }^{17}$ Another common problem encountered by surgeons using an open dorsal or dorsolateral approach is the limited visualization of the ventral dura. Dorsal thorascopic techniques overcome this problem through the employment of an angled endoscope that can aid in ventral visualization.

One of the most common thoracic spine pathologies treated via dorsal endoscopic approaches is disk herniation. ${ }^{16}$ In dorsal thorascopic approaches, dorsolateral (or posterolateral) access via the transforaminal approach results in the least morbidity of any method of dorsal thoracic spine surgery. ${ }^{16}$ This reduction in morbidity is achieved through small incisions, minimal muscle dissection, and the ability to complete these surgeries under local anesthesia. For this approach, the patient is placed in the prone 
position and is prepped and draped in standard sterile fashion. The endoscopic entry point is outlined by drawing a line from the midpedicular annulus to the lateral facet with extension of that line onto the skin. With fluoroscopic guidance, a discography needle is inserted to target and image the disk space of interest. Upon completion of discography, the needle is replaced by sequentially enlarging reamers used to dilate the neural foramen until endoscopic forceps can be successfully introduced to perform the discectomy and decompression under endoscopic visualization. ${ }^{16}$ Similarly to the transforaminal approach, the oblique paraspinal approach using tubular microendoscopy is commonly used for discectomy in the case of disk herniation. This approach uses a trajectory that is less oblique than the transforaminal approach and designates the superior border of the caudal transverse process as the endoscopic entry point. ${ }^{16}$ It is particularly beneficial in the discectomy of sequestered and calcified herniated disks. Additionally, the authors note that the ribs often limit access to medially located herniations in the midthoracic spine. The rib curvature can interfere with the tilting of the endoscope laterally, which makes it difficult to gain access to the medial part of the disc space. Therefore, when using this approach, it may be most suited for the lower thoracic spine. ${ }^{15}$ Osman and Marsolais $^{18}$ completed a cadaveric study on the feasibility of a posterolateral approach for arthroscopic thoracic discectomy, and they noted that a more lateral, shallower approach gives the surgeon better access to the epidural space, but a more posterior or steep approach would allow better access to lateral herniation.

Jho ${ }^{19,20}$ described the percutaneous endoscopic transpedicular thoracic discectomy from a posterolateral approach. The limitation, however, is that this cannot give the surgeon a direct visualization of the ventral spinal cord. Therefore, the author employed a $70^{\circ}$ angled endoscope to visualize the ventral cord. In their technique, they removed the ipsilateral facet joint as well as the upper part of the pedicle, which created exposure of the neural foramen, intervertebral disc, and the upper aspect of the pedicle leading to the vertebral bodies. The herniated discs were removed laterally, and a cavity was created under the operating microscope. Then a $70^{\circ}$ angled endoscope to visualize the ventral cord directly was used. One of the major drawbacks to this technique, however, was the reversed perception of the surgical field. ${ }^{19,20}$ Choi et $\mathrm{al}^{21}$ provided further evidence on the safety, as well as efficacy, of the percutaneous endoscopic thoracic discectomy from a posterolateral approach. In this case, the authors removed the herniated disc through the thoracic intervertebral foramen after foraminoplasty by cutting away the lateral and inferior aspects of the superior facet with a round cutter. The authors found that this approach had positive outcomes based on visual analog scale and Oswestry Disability Index. Nie and $\mathrm{Liu}^{22}$ described a case series using endoscopic transforaminal thoracic foraminotomy and discectomy for the treatment ofthoracic disc herniation, employing a technique similar to that of Choi et al. In this series, a diverse range of disc levels were treated, from T5 to T6, and T12 to L1. All patients had immediate pain relief, and most were satisfied postoperatively. Wagner et $\mathrm{al}^{23}$ described a percutaneous transforaminal thoracic endoscopic foraminoplasty procedure and compared this to thoracic microendoscopic discetomy. In the transforaminal procedure, under fluoroscopy, the needle trajectory ought to cross the isthmus but not the area under the superior pedicle. The target is the superior end plate of the inferior vertebral body. Whereas in thoracic microendoscopic discetomy, the trajectory is direct and targets the superior aspect of the caudal transverse process. $^{22}$

A recent retrospective study by Xiaobing et $\mathrm{al}^{24}$ was done on 14 patients with thoracic spinal stenosis caused by TDH, ossification of the ligamentum flavum, or ossification of the posterior longitudinal ligament. Treatment was done with " $U$ route" percutaneous transforaminal endoscopic thoracic discectomy, and the authors concluded that this is a feasible alternative to the threat of thoracic spinal stenosis. ${ }^{23}$ Finally, Telfeian et $\mathrm{al}^{25}$ described the unique case of performing transforaminal endoscopic spine surgery at the thoracolumbar junction. They described 3 technical challenges, which include proximity of the kidney, rib, and the thecal sac/spinal canal diameter ratio. A safe corridor was mapped on preoperative axial MRI in order to avoid the kidney and rib. Additionally, particular consideration was given to the thecal sac/spinal canal diameter ratio, referencing studies that have demonstrated differences in the interpedicular distance (transverse diameter of the spinal canal) at L1 $(22 \mathrm{~mm})$ versus L5 $(30 \mathrm{~mm})$. When comparing the transforaminal targeting at 
L5, the target is usually the medial pedicle wall on anterior-posterior fluoroscopy, but if this is applied to T12 to L1, then the endoscope would enter the thecal sac. Therefore, the trajectory of the needle must be planned to enter the disc space at the midpedicle line. ${ }^{25}$

Regarding preoperative planning for treating TDH with a transforaminal endoscopic approach, a safe corridor should be mapped out on preoperative axial MRI in order to avoid the lungs and ribs. Additionally, attention should be given to the thecal sac/spinal canal diameter ratio. The trajectory for the needle in transforaminal targeting aims to increase access to the ventral aspect of the thecal sac without causing injury to the dura. The authors note that a more lateral approach with a smaller tubular retractor helps in accessing ventral disease but also mention that a significant amount of disc pathology seen in the thoracic spine is calcified and would be less effective in these cases. ${ }^{22}$

Similar to ventral approaches, dorsal or dorsolateral endoscopic approaches to the thoracic spine provide the opportunity to achieve successful surgical outcomes with minimal complications. The posterolateral approach would be best suited for soft, lateral disc herniation. In addition, it may be best for patients who are more muscular, and would require longer incision, or more muscle dissection with the standard approaches. ${ }^{26}$ As mentioned previously, a more detailed comparison of outcomes between open and endoscopic approaches to thoracic spine surgery will be discussed in later sections.

\section{ANATOMIC CONSIDERATIONS}

There are various anatomic considerations that need to be made depending on the route of endoscopic approach. One major factor is kyphosis in the thoracic spine. With ventral subarachnoid space measuring 1 to $3 \mathrm{~mm}$, and the dorsal subarachnoid space at 2 to $6 \mathrm{~mm}$, ventral approach leaves little room for error. ${ }^{27}$ The anterior mobility of the spinal cord upon placing a patient in the prone position lessens ventral subarachnoid space. Therefore, patients must be placed in the lateral position during ventral entry to reduce risk of spinal cord damage. ${ }^{27}$ Anatomically, the intercostal muscles are separated to get to the retropleural space. The next layer resected is the rib head and pedicle to arrive at the epidural space, thus exposing the disc. ${ }^{27}$
Due to angle of entry, there is a risk of opening the thoracic and pleural cavities. ${ }^{3}$

Endoscopic approach from the dorsolateral route prevents a need for bone resection, and it occurs directly through the window of the intervertebral foramen. Entering posterolaterally, a foraminotomy is performed to enlarge cannula access to the inner foraminal zone. Both the articular facet and the rib head are undercut to enlarge the foramen, thus exposing the epidural space and exiting spinal nerve roots. ${ }^{27}$ However, there is an increased risk of perforation and visceral damage to the lungs along with vascular damage to the inferior vena cava and thoracic aorta. ${ }^{27}$ Patients should be placed in the lateral position on the contralateral side of the lesion to increase available subarachnoid space and decrease risk of spinal cord damage. ${ }^{27}$ Dorsal endoscopic approach starts with bone resection of the descending facet and bottom spinous process to arrive at the ligamentum flavum. The next structure under is epidural fat that is resected to arrive at the neural tissue, where the herniation can be identified. ${ }^{4}$ Through the dorsal approach, the patient is best placed in a prone position because of anterior spinal cord movement, and thus enlargement of subarachnoid space. ${ }^{3}$

\section{ANESTHESIA FOR THORACIC SPINE SURGERY}

There are a few key factors to consider when screening and selecting for optimal anesthesia control during thoracic endoscopic spinal surgeries. Thoracic endoscopic spinal surgery can involve general anesthesia or regional/local anesthesia. It is important to consider the risks versus the benefits of these 2 options for anesthesia. With the use of regional or local anesthesia, outpatient surgery or same-day surgery is feasible because the regional anesthesia allows for a reduced operative time from induction to extubation, as well as a shorter hospital stay. ${ }^{28}$ Also, procedure time may be shorter using regional anesthesia rather than general anesthesia mostly because of a shorter interval between induction and incision but also following wound closure because there is no need to await recovery from the neuromuscular blockade. ${ }^{29}$

Most thoracic endoscopic spinal surgeries rely on the use of regional anesthesia due to its benefits. The absolute major contraindications to regional anesthesia include lack of patient consent and elevated intracranial pressure, primarily due to intracranial 
mass or infection at the procedure site. ${ }^{30}$ The relative contraindications to regional anesthesia to consider include preexisting neurologic disease, hypovolemia, hypotension, thrombocytopenia or other coagulopathies, severe mitral or aortic stenosis, and left ventricular outflow obstruction, as seen in hypertrophic obstructive cardiomyopathy. ${ }^{30}$ It is also important to note that the incidence of postdural puncture headache following regional anesthesia seems to be extremely low because of the speculation that surgical bleeding in the area of the dural puncture site serves as a natural blood patch. $^{30}$

The regional and local anesthesia used in thoracic endoscopic surgeries typically involves first administering a sedative, such as midazolam IV (1-2mg) and fentanyl IV (usual initial dose range: $0.5-2 \mu \mathrm{g} / \mathrm{kg}$, administered in incremental boluses of $25-50 \mu \mathrm{g}$, titrated to effect). Following sedative administration, anesthesia is obtained through $0.5 \%$ bupivacaine without epinephrine during 5 to 10 seconds, with the dose modified according to patient factors (eg, height, age, body mass index). ${ }^{32}$ After the regional anesthesia is administered appropriately, the patient is positioned for surgery.

\section{PATIENT POSITIONING}

Proper patient positioning for thoracic spine surgery is important for optimal operating conditions. During spine surgery, patients are often placed in nonphysiologic conditions, which may lead to complications. ${ }^{33}$ Among complications, it is important to be aware of peripheral nerve injury and postoperative visual loss because these are rare complications related to patient positioning during spine surgery that result in significant patient disability and loss of function. ${ }^{33}$ In addition to these rare complications of prone positioning, it is important to be aware of increased intra-abdominal pressure when patients are placed in the prone position. It has been shown that reduced intraabdominal pressure reduces blood loss during surgery due to the reduction of inferior vena cava pressure. ${ }^{34}$ Using prone positioning during thoracic spinal surgery can result in a reduction of cardiac index, which has been attributed to a reduction in venous return to the heart and a reduced left ventricular compliance as a result of increased intrathoracic pressure. ${ }^{35}$

\section{EQUIPMENT}

With refinement of the endoscope in the 1990s into the early 2000 s, thoracoscopic spine surgery has vastly evolved in intervention in various thoracic spine pathologies. ${ }^{36}$ Common rigid thoracoscopes include a $0^{\circ}$ angled scope, a $30^{\circ}$ oblique angled scope, and a $45^{\circ}$ oblique angled scope. ${ }^{37}$ Each type of thoracoscope provides up to $120^{\circ}$ direction of view which can be rotated circumferentially using the turn dial on the thoracoscope handle. ${ }^{37}$ The type of oblique angled scope used depends on surgeon preference and the thoracoscopic approach to the involved vertebrae. Fiberoptic light transmission is directly connected from a light source to the scope to facilitate clear images onto a viewing monitor. Improvements in the thoracoscope have included higher resolution for clearer images and innovative accessory devices to reduce the strain on the surgeon. Although thoracoscopy has reduced the invasiveness of certain thoracic spine surgeries, the learning curve of spatial orientation and depth perception can be challenging for novice surgeons. The integration of an image guidance system with thoracoscopy offers intraoperative $\mathrm{CT}$ reconstruction of the patient's bony anatomy to guide placement of a drill guide, verify orientation of implants, and evaluate bony resection and decompression. ${ }^{38}$ Although the benefits of an image guidance system can be multifactorial, the reliability is dependent on minimal error associated with the CT scan itself or the automated reconstruction technology. ${ }^{38}$ The future of thoracoscopic spine surgery is trending toward robotic-assisted MIS with an emphasis on improvement of both the accuracy and the trajectory of implant placement to ensure minimal complication risk and effective approach. $^{39}$

\section{RESULTS/OUTCOMES}

Since the late 1990s, the literature has continued to grow, with publications reporting on the feasibility and success of minimally invasive thorascopic procedures for a variety of spinal pathologies. The earliest large-sample report of patients receiving thoracic spine surgery via endoscopic methods was published by Han et $\mathrm{al}^{14}$ in 2002, in which the authors reviewed 241 thorascopic procedures performed between January 1994 and January 2000. Between 164 sympathectomies, 60 discectomies, 5 tumor resections, 8 corpectomies, 2 vertebral body 
biopsies, and 2 spinal deformity corrections, the success rates for all types of procedures ranged between $98 \%$ and $100 \%$, with no observed operative mortality and a very low rate of morbidity (minor pulmonary complications, superficial wound infections, and costovertebral joint pain in less than $5 \%$ of patients) that was favorable compared with open surgical approaches. In 2006, Ringel et $\mathrm{al}^{40}$ published a retrospective review of 115 thoracic (and lumbar) fixation procedures in 104 patients between May 2002 and May 2005 using percutaneous minimally invasive procedures. This review also reported high success rates, with postoperative CT imaging demonstrating $87 \%$ of screw positions as "good," $10 \%$ as "acceptable," and 3\% as "unacceptable," requiring a total of 11 revisions for misplaced screws or loosening of anchor bolts. There were no reports of patients experiencing new neurological deficits, surgery-related morbidity, or mortality.

To build on these initial data demonstrating the success of MISS, there have been a few cohort studies comparing outcomes between MISS and traditional open surgical techniques. For example, in 2009 Lonner et $\mathrm{al}^{41}$ published a matched-pair analysis of 34 consecutive adolescent patients (in 17 pairs) who were randomized to receive either VATS or open posterior spinal fusion with thoracic pedicle screws for treatment of idiopathic structural scoliosis. The results of this trial demonstrated that the group receiving VATS had significantly increased operative duration compared with posterior spinal fusion (which falls in line with the increased level of surgical difficulty of VATS), but also had reduced intraoperative blood loss. Between the 2 groups, there were no statistically significant differences between blood-product transfusion rate, length of postoperative hospital stay duration, or qualitative results of the Scoliosis Research Society-22 (SRS22) outcome questionnaire assessing pain, selfimage, function, mental health, and satisfaction. Both groups also experienced similar improvements from baseline in terms of pulmonary function at the 24-month postoperative follow-up time point. In 2014, Lee et $\mathrm{al}^{42}$ published a retrospective cohort study of 40 patients comparing MISS with traditional anterior spinal surgery (TASS) for the treatment of thoracic (or lumbar) infectious spondylitis (23 in the MISS group, 17 in the TASS group). Their results indicated that in comparison to patients undergoing TASS, patients receiving MISS experienced decreased mean estimated blood loss and packed red blood cell transfusion intraoperatively, decreased postoperative tube drainage, a decreased need for postoperative intensive care unit care, and a decreased length of hospital stay. Although surgical success was achieved in $97 \%$ of all patients with no differences between groups, the MISS group experienced a complication rate that amounted to one-third of the rate of complications in the TASS group, with no major complications in the MISS group compared with 4 major complications in the TASS group. Lastly, the aforementioned publication by Ruetten et $\mathrm{al}^{3}$ reviewed a series of 55 fully endoscopic uniportal technique decompression procedures between 2009 and 2015 that used either an interlaminar, extraforaminal, or transthoracic retropleural approaches for thoracic disc herniation or stenosis. Follow-up data at the 18-month postoperative mark illustrated sufficient decompression in nearly all patients, with only 1 patient requiring revision due to secondary bleeding. Only 1 patient exhibited persistent deterioration of their preoperative myelopathy, whereas all other patients experienced regression or improvement of their symptoms. Overall, although the literature on MISS using a posterior or posteriolateral approach continues to grow, these series of reviews and trials illustrate the success and high rate of positive patient outcomes that can be had with minimally invasive endoscopic thoracic spine surgery techniques compared with open approaches that tend to carry a higher rate of intraoperative and postoperative complications.

\section{AVOIDANCE OF COMPLICATIONS}

Complications in MISS are mainly elevated in surgeons who lack familiarity with these techniques. Therefore, it is important to ensure that MISS is performed by surgeons with extensive training in the field. ${ }^{43}$ Another method of reducing complications is to minimize the use of transthoracic approaches to access the thoracic spine. While the transthoracic approach may provide better visualization of the surgical field, it is associated with myriad postoperative pulmonary complications, which include atelectasis, pneumonitis, pleuritis, intercostal neuralgia, and the need for postoperative chest tube placement. $^{15}$

Posterolateral approaches on the other hand, reduce the risk of pulmonary complications but may not provide equal access to the surgical site. 
However, innovations in posterolateral approaches like the retropleural approach by Kasliwal and Deutsch ${ }^{44}$ enable equally good access to the surgical field without increasing the risk of complications. Hence, promoting the use and technologic development of more posterolateral approaches is crucial to lowering MISS complications in the future. ${ }^{15}$

\section{CONCLUSION}

It has been established that open approaches to access the thoracic spine not only increase tissue trauma but also prolong patient recovery time. Contrastingly, MISS techniques reduce tissue trauma and patient recovery time. Among MISS approaches, ventral or transthoracic approaches tend to increase the probability of postoperative pulmonary complications. Therefore, emphasizing the use of posterolateral procedures may prove to be the best method of lowering complication rates in MISS. However, it is important to note that ventral and transthoracic approaches often allow better visualization of the surgical field. Hence, using the ventral approach may be more effective when visualization is a vital aspect of the surgical procedure. Nonetheless, recent developments in lateral approaches like the retropleural approach provide equally good visualization of the anterior spine as ventral approaches, without the increased risk of pulmonary complications. Accordingly, it is crucial that further training and innovations in posterolateral MISS approaches for the thoracic spine are promoted in the future.

\section{REFERENCES}

1. Ahn Y. Current techniques of endoscopic decompression in spine surgery. Ann Transl Med. 2019;7(suppl 5):S169-S169.

2. Telfeian AE, Veeravagu A, Oyelese AA, Gokaslan ZL. A brief history of endoscopic spine surgery. Neurosurg Focus. 2016;40(2):E2.

3. Ruetten S, Hahn P, Oezdemir S, et al. Full-endoscopic uniportal decompression in disc herniations and stenosis of the thoracic spine using the interlaminar, extraforaminal, or transthoracic retropleural approach. $J$ Neurosurg Spine. 2018;29(2):157-168.

4. Choi G, Pophale CS, Patel B, Uniyal P. Endoscopic spine surgery. J Korean Neurosurg Soc. 2017;60(5):485-497.

5. Lin CY, Chang CC, Chen YJ, et al. New strategy for minimally invasive endoscopic surgery to treat infectious spondylodiscitis in the thoracolumbar spine. Pain Physician. 2019;22(3):281-293.

6. Bae J, Chachan S, Shin SH, Lee SH. Percutaneous endoscopic thoracic discectomy in the upper and midthoracic spine: a technical note. Neurospine. 2019;16(1):148-153.
7. Abuzayed B, Tuna Y, Gazioglu N. Thoracoscopic anatomy and approaches of the anterior thoracic spine: cadaver study. Surg Radiol Anat. 2012;34(6):539-549.

8. Birkenmaier C, Chiu J, Fontanella A, Leu H, Ruetten S. Guidelines for endoscopic spinal surgery. Ortop Travmatol Protez. 2014;87-95.

9. Cofano F, Di Perna G, Marengo N, et al. Transpedicular 3D endoscope-assisted thoracic corpectomy for separation surgery in spinal metastases: feasibility of the technique and preliminary results of a promising experience. Neurosurg Rev. 2020;43(1):351-360.

10. Visocchi M, Masferrer R, Sonntag VK, Dickman CA. Thoracoscopic approaches to the thoracic spine. Acta Neurochir (Wien). 1998;140(8):737-743; discussion 743-734.

11. Rosenthal D. Endoscopic approaches to the thoracic spine. Eur Spine J. 2000;9(suppl 1:S8-S16.

12. De Giacomo T, Francioni F, Diso D, et al. Anterior approach to the thoracic spine. Interact Cardiovasc Thorac Surg. 2011;12(5):692-695.

13. Kan P, Schmidt MH. Minimally invasive thoracoscopic approach for anterior decompression and stabilization of metastatic spine disease. Neurosurg Focus. 2008;25(2):E8.

14. Han PP, Kenny K, Dickman CA. Thoracoscopic approaches to the thoracic spine: experience with 241 surgical procedures. Neurosurgery. 2002;51(5 suppl):S88-S95.

15. Sharma SB, Kim JS. A review of minimally invasive surgical techniques for the management of thoracic disc herniations. Neurospine. 2019;16(1):24-33.

16. Smith JS, Ogden AT, Fessler RG. Minimally invasive posterior thoracic fusion. Neurosurg Focus. 2008;25(2):E9.

17. Hasan S, Hartl R, Hofstetter CP. The benefit zone of full-endoscopic spine surgery. J Spine Surg. 2019;5(suppl 1):S41-S56.

18. Osman SG, Marsolais EB. Posterolateral arthroscopic discectomies of the thoracic and lumbar spine. Clin Orthop Relat Res. 1994;(304):122-129.

19. Jho HD. Endoscopic transpedicular thoracic discectomy. J Neurosurg. 1999;91(2 suppl):151-156.

20. Jho HD. Endoscopic microscopic transpedicular thoracic discectomy: technical note. J Neurosurg. 1997;87(1):125-129.

21. Choi KY, Eun SS, Lee SH, Lee HY. Percutaneous endoscopic thoracic discectomy; transforaminal approach. Minim Invasive Neurosurg. 2010;53(1):25-28.

22. Nie H., Liu K. (2013). Endoscopic transforaminal thoracic foraminotomy and discectomy for the treatment of thoracic disc herniation. Minimally Invasive Surgery, 2013;2013:264105. doi: 10.1155/2013/264105

23. Wagner R, Telfeian AE, Iprenburg M, et al. Transforaminal endoscopic foraminoplasty and discectomy for the treatment of a thoracic disc herniation. World Neurosurg. 2016;90:194-198.

24. Xiaobing Z, Xingchen L, Honggang Z, et al. "U" route transforaminal percutaneous endoscopic thoracic discectomy as a new treatment for thoracic spinal stenosis. Int Orthop. 2019;43(4):825-832.

25. Telfeian AE, Jasper GP, Oyelese AA, Gokaslan ZL. Technical considerations in transforaminal endoscopic spine surgery at the thoracolumbar junction: report of 3 cases. Neurosurg Focus. 2016;40(2):E9.

26. Isaacs RE, Podichetty VK, Santiago P, et al. Minimally invasive microendoscopy-assisted transforaminal lumbar inter- 
body fusion with instrumentation. $J$ Neurosurg Spine. 2005;3(2):98-105.

27. Shimada S, Tamaki N. Assessment of safety and feasibility of spinal endoscope in the thoracic and lumbar region: a cadaveric study. Kobe J Med Sci. 2001;47(6):263-272.

28. Ahn Y. Current techniques of endoscopic decompression in spine surgery. Ann Transl Med. 2019;7(suppl 5):S169.

29. McLain RF, Tetzlaff JE, Bell GR, Uwe-Lewandrowski K, Yoon HJ, Rana M. Microdiscectomy: spinal anesthesia offers optimal results in general patient population. J Surg Orthop Adv. 2007;16(1):5-11.

30. Olawin AM. Spinal anesthesia. In: StatPearls. Treasure Island, FL: StatPerls Publishing. 2020.

31. De Rojas JO, Syre P, Welch WC. Regional anesthesia versus general anesthesia for surgery on the lumbar spine: a review of the modern literature. Clin Neurol Neurosurg. 2014;119:39-43.

32. Brown MJ. Anesthesia for elective spine surgery in adults. https://www.uptodate.com/contents/anesthesia-for-elec tive-spine-surgery-in-adults? search $=$ anesthesia $\% 20$ for $\% 20$ tho racic $\% 20$ spine $\% 20$ surgery\&source=search_result\&selected Ti tle $=1 \sim 150 \&$ usage_type $=$ default\&display_rank $=1$. Accessed April 9, 2020.

33. Kamel I, Barnette R. Positioning patients for spine surgery: avoiding uncommon position-related complications. World J Orthop. 2014;5(4):425-443.

34. Lee TC, Yang LC, Chen HJ. Effect of patient position and hypotensive anesthesia on inferior vena caval pressure. Spine (Phila Pa 1976). 1998;23(8):941-947; discussion 947-948.

35. Sudheer PS, Logan SW, Ateleanu B, Hall JE. Haemodynamic effects of the prone position: a comparison of propofol total intravenous and inhalation anaesthesia. Anaesthesia. 2006;61(2):138-141.

36. Baron EM, Levene HB, Heller JE, Jallo JI, Loftus CM, Dominique DA. Neuroendoscopy for spinal disorders: a brief review. Neurosurg Focus. 2005;19(6):E5.

37. Storz K. The world of endoscopy. In: Thorax. Tuttlingen, Germany: Karl Storz SE \& Co; 2011.

38. Johnson JP, Drazin D, King WA, Kim TT. Imageguided navigation and video-assisted thoracoscopic spine surgery: the second generation. Neurosurg Focus. 2014;36(3):E8.

39. Yeung AT. Robotics in the MIS spine surgery arena: a new role to advance the adoption of endoscopic surgery as the least invasive spine surgery procedure. J Spine. 2017;6(3). doi:10.4172/2165-7939.1000374

40. Ringel F, Stoffel M, Stüer C, Meyer B. Minimally invasive transmuscular pedicle screw fixation of the thoracic and lumbar spine. Neurosurgery. 2006;59(suppl 4):ONS361ONS367.

41. Lonner BS, Auerbach JD, Estreicher M, Milby AH, Kean KE. Video-assisted thoracoscopic spinal fusion compared with posterior spinal fusion with thoracic pedicle screws for thoracic adolescent idiopathic scoliosis. J Bone Joint Surg. 2009;91(2):398-408.

42. Lee CY, Huang TJ, Li YY, Cheng CC, Wu MH. Comparison of minimal access and traditional anterior spinal surgery in managing infectious spondylitis: a minimum 2-year follow-up. Spine J. 2014;14(7):1099-1105.

43. Derman PB, Phillips FM. Complication avoidance in minimally invasive spinal surgery. J Spine Surg. 2019;5(suppl 1):S57-S67.

44. Kasliwal MK, Deutsch H. Minimally invasive retropleural approach for central thoracic disc herniation. Minim Invasive Neurosurg. 2011;54:167-171.

Disclosures and COI: The authors report no conflict of interest concerning the materials or methods used in this study or the findings specified in this paper. The authors have not received any funding for this work from any organization.

Corresponding Author: Brian Fiani, DO, Desert Regional Medical Center, 1180 North Indian Canyon Dr, Ste 214 W, Palm Springs, CA 92262. Phone: (760) 416-4511; Email: bfiani@outlook.com.

Published 11 November 2020

This manuscript is generously published free of charge by ISASS, the International Society for the Advancement of Spine Surgery. Copyright (c) 2020 ISASS. To see more or order reprints or permissions, see http://ijssurgery.com. 